\title{
Fatigue Process Evaluation of Ultrasonic Fatigue Testing in High Strength Steel Analyzed by Acoustic Emission and Non-Linear Ultrasonic*
}

\author{
Mitsuharu Shiwa $^{1}$, Yoshiyuki Furuya ${ }^{1}$, Hisashi Yamawaki ${ }^{1},{\text { Kaita } \text { Ito }^{2} \text { and Manabu Enoki }}^{2}$ \\ ${ }^{1}$ National Institute for Materials Science, Tsukuba 305-0047, Japan \\ ${ }^{2}$ Department of Materials Engineering, School of Engineering, The University of Tokyo, Tokyo 113-8656, Japan
}

\begin{abstract}
Non-linear ultrasonic and acoustic emission (AE) signals during ultrasonic fatigue testing were analyzed by using Laser Doppler Vibrometer (LDV) and continuous AE waveform analysis system (1 MHz/12 bit). Notched specimens of a high strength low alloy steel were prepared for the ultrasonic fatigue test with exciting vibration frequency of $20 \mathrm{kHz}$. The detected surface velocity was longitudinal direction at the end of specimen with frequency range from $200 \mathrm{~Hz}$ to $500 \mathrm{kHz}$. During the waveform monitoring of the fatigue test, a distorted exciting waveform was observed in final stage of the test. Then the burst type noise mixed with the distorted exciting waveform was obtained just before the final failure in the case of failure specimens. Contrary, the distorted exciting waveform and AE were not observed in the case of non-failure specimens. AE signal and upper harmonics of exciting frequency were analyzed by the FFT method. As the result, after the intensity of 2 nd and 3rd harmonics increased rapidly, AE events were detected continuously in the case of failure specimens. It can be concluded that nonlinear ultrasonic and AE analysis were effective monitoring tool for fatigue damage progression. [doi:10.2320/matertrans.M2010074]
\end{abstract}

(Received February 26, 2010; Accepted May 17, 2010; Published June 30, 2010)

Keywords: ultrasonic fatigue testing, high strength low alloy steel, nonlinear ultrasonic, acoustic emission

\section{Introduction}

Ultrasonic fatigue testing technique was applied to a gigacycle fatigue testing of the high-strength steel with a tensile strength of $1200 \mathrm{MPa}$ in NIMS. The typically exciting frequency of the ultrasonic fatigue testing is $20 \mathrm{kHz}$. Since the giga-cycle fatigue was caused by internal non-metallic inclusions or matrixes leading to fish-eye fractures, it is possible to evaluate the size of inclusion in a short time with this technique. ${ }^{1)}$ In the SUP7 spring steel, the observed fracture surface of giga-cycle fatigue testing in optical dark areas (ODAs) was the fish-eye fracture formed around internal inclusions of $\mathrm{Al}_{2} \mathrm{O}_{3}$ with an approximate diameter of $30 \mu \mathrm{m}$. It is reported that this fish-eye fracture is not a simple hydrogen embrittlement process during the ultrasonic fatigue testing. ${ }^{2)}$ Usually, a fatigue crack growth process of the plane specimen has four stages, which are crack nucleation, crack initiation, crack arrest and crack propagation in sequence. Because most of the fatigue life has been spent on the occurrence of crack nucleation, the in-situ observation technique is necessary to estimate the crack nucleation process during fatigue testing.

In the typical non-destructive evaluation techniques of fatigue damage, the parameters of velocity and attenuation for the ultrasonic wave were often adopted. By the theory of Granato-Lücke, it can be known that the changes of those parameters are determined by the dislocation length and density. Recently, Cantrell ${ }^{3,4)}$ reported that there is a method based on nonlinear acoustics, which offers a quantitative assessment of the plastic deformation in wavy slip metals from the initial state to fatigue damage. In addition, it shows that the nonlinear ultrasonic, such as the sub harmonic, is mainly attributed to the crack surface nucleation, ${ }^{5)}$ and the acoustic emission (AE) is due to the micro-crack generation. $^{6 \text { ) }}$

*This Paper was Originally Published in Japanese in J. Japan Inst. Metals 73 (2009) 250-210.

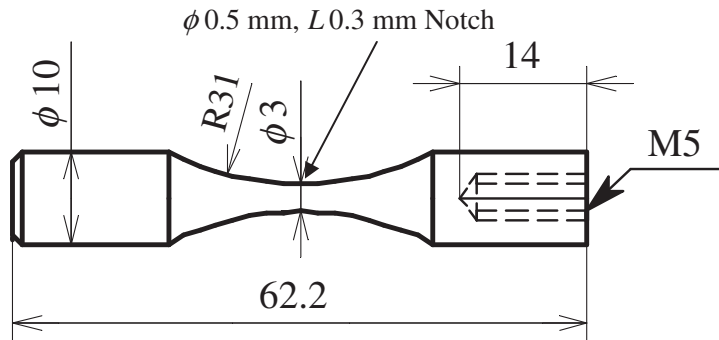

Fig. 1 Geometry of specimen.

In this work, the notched specimens with a clear progress route of the crack were used. Nonlinear ultrasonic and AE signals were measured simultaneously by a wide band Laser Doppler Vibrometer (LDV), which can detect the surface vibration of specimen directly. ${ }^{7)}$

\section{Experimental Procedure}

The JIS SCM440 of a low-alloy steel was used as the principal alloy. The present specimens were prepared by the sequential heat treatments (quenched at $1153 \mathrm{~K}$ for $30 \mathrm{~min}$ and tempered at $473 \mathrm{~K}$ for $60 \mathrm{~min}$ ), which leads to the internal fracture in the material structure. ${ }^{2)}$ The Vickers hardness of specimens was from HV 550 to HV 560. Figure 1 shows the geometry of the specimen. At the center of specimen, an artificial notch with $0.3 \mathrm{~mm}$ in diameter and $0.3 \mathrm{~mm}$ in depth was generated by EDM.

The fatigue testing was conducted on an ultrasonic fatigue testing machine with an excitation frequency of $20 \mathrm{kHz}$. The applied stress of the fatigue testing varied from $180 \mathrm{MPa}$ to $420 \mathrm{MPa}$ with the air cooling, which can be seen in Fig. 2.

A laser Doppler vibrometer (Graphtec, AT0042 + AT3600L) was used to the experiment. The measured frequency range was from $200 \mathrm{~Hz}$ to $1.3 \mathrm{MHz}$ with a high pass filter $200 \mathrm{~Hz}$ and $24 \mathrm{~dB} /$ oct. cutting, and the measurement range was $1 \mathrm{~V} / \mathrm{m} / \mathrm{s}$. The vibration on the specimen was measured by introducing the laser beam into the bottom of 


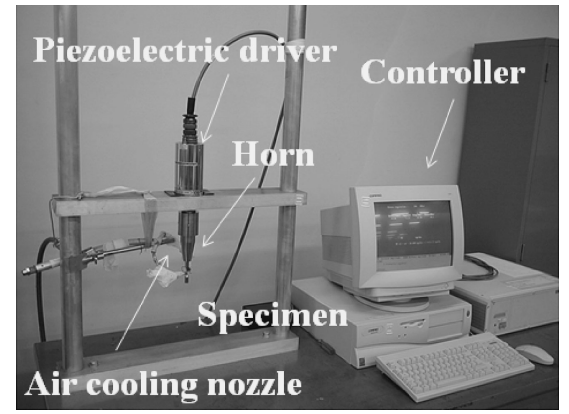

Fig. 2 Ultrasonic fatigue testing machine.

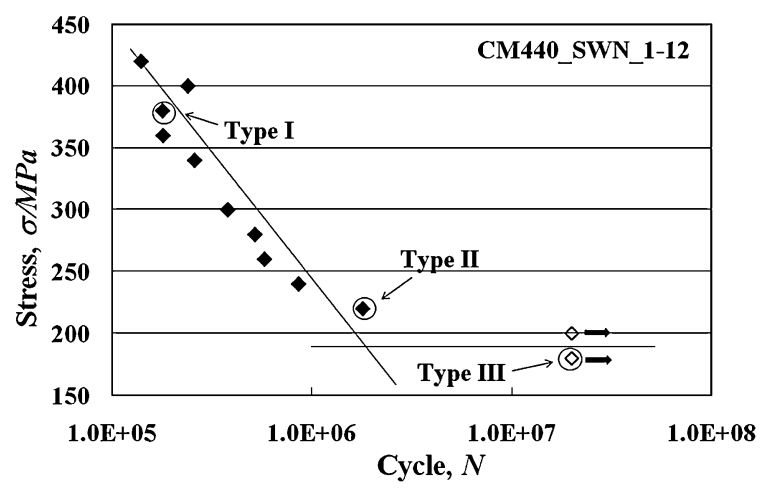

Fig. $3 \mathrm{~S} / \mathrm{N}$ curve of Notched SCM440.

specimen that was a free edge of longitudinal vibration. Meanwhile, the continuous waveform memory (CWM) was used to analyze the intensity of harmonics and the event count of acoustic emission. ${ }^{8)}$ The sampling frequency of the $\mathrm{A} / \mathrm{D}$ converter was $1 \mathrm{MHz}$ and the resolution was $12 \mathrm{bit}$.

\section{Experimental Results}

\subsection{Condition of frequency analysis}

Figure 3 gives the $\mathrm{S} / \mathrm{N}$ curves of specimens. The fatigue limit was $190 \mathrm{MPa}$. Then three stages of stress step, such as the low cycle number of fatigue failure (380 MPa: Type I), the high cycle number of fatigue failure (220 MPa: Type II) and the non-breaking (180 MPa: Type III) were analyzed by a frequency domain.

In present ultrasonic fatigue testing, the resonant frequency and the excitation magnitude of specimen were controlled by the feedback process to keep the preset stress. It has to analyze the phenomena of them at first. In the frequency domain analysis such as FFT, the frequency resolution and the time resolution are incompatible. Then, the relationship between the frequency resolution and the time step which means a packet of fatigue cycles in this experiment was analyzed. For example, when the frequency resolution of $1 \mathrm{~Hz}$ is obtained at $1 \mu \mathrm{s}$ sampled, 1 mega samplings are needed. To obtain the frequency resolution of $1 \mathrm{~Hz}$, each $2 \times 10^{4}$ cycle was performed.

Different time steps of the frequency resolution of $100 \mathrm{~Hz}$ and $1 \mathrm{~Hz}$ in the middle stage of the test of Type II are shown in Fig. 4. Figures 4(a), (b), (c) represent the frequency resolution of $100 \mathrm{~Hz}$ and the time step of $10 \mathrm{~ms}$ (200 cycles), $1 \mathrm{~Hz}$ and $100 \mathrm{~ms}$ (2000 cycles) and $1 \mathrm{~Hz}$ and $10 \mathrm{~ms}(200$
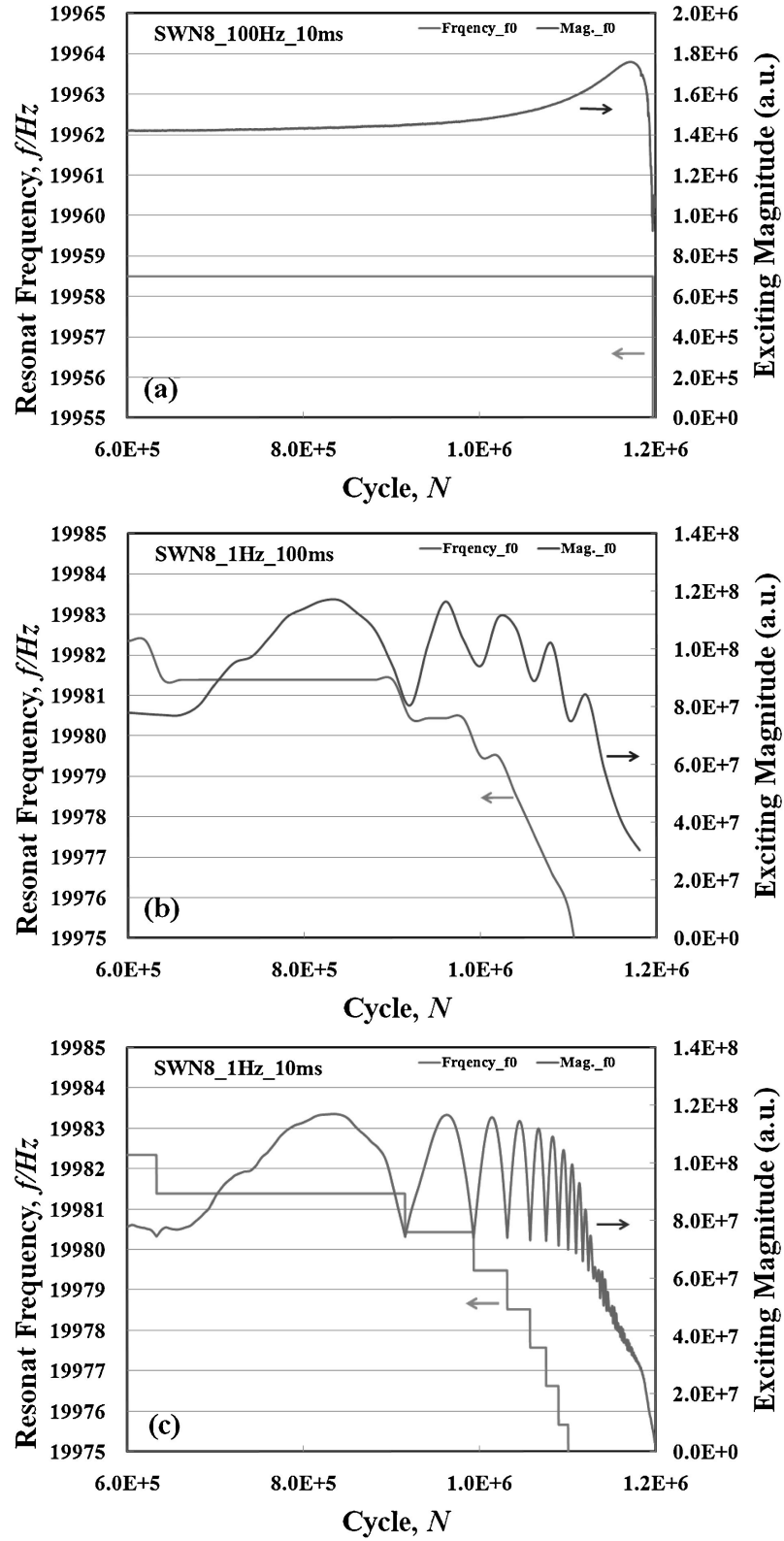

Fig. 4 Resonant frequency and magnitude during ultrasonic fatigue testing of Type I with different FFT conditions, $100 \mathrm{~Hz} / 10 \mathrm{~ms}$ steps (a), $1 \mathrm{~Hz} /$ $100 \mathrm{~ms}$ steps (b), $1 \mathrm{~Hz} / 10 \mathrm{~ms}$ steps (c).

cycles), respectively. The shift of resonant frequency was clearly observed with the frequency resolution of $1 \mathrm{~Hz}$. Meanwhile, the shift of excitation magnitude was clearly observed with the time step of $10 \mathrm{~ms}$. When the similar analyses were carried out for Type II and III, the similar results of Type I was observed in Type II of the broken specimen. However, the comparable results in Type III of unbroken specimen were obtained in each time step. Besides, the time step of FFT was selected $10 \mathrm{~ms}$ in order to analyze nonlinear ultrasonic of Type I and Type II, and that of Type III was selected $1000 \mathrm{~ms}$ with a frequency resolution of $1 \mathrm{~Hz}$.

\subsection{Non-linear ultrasonic analysis (sub-harmonic and harmonics)}

Figure 5 shows the relationship between the exciting magnitude and the nonlinear ultrasonic of Type I (a), Type II 

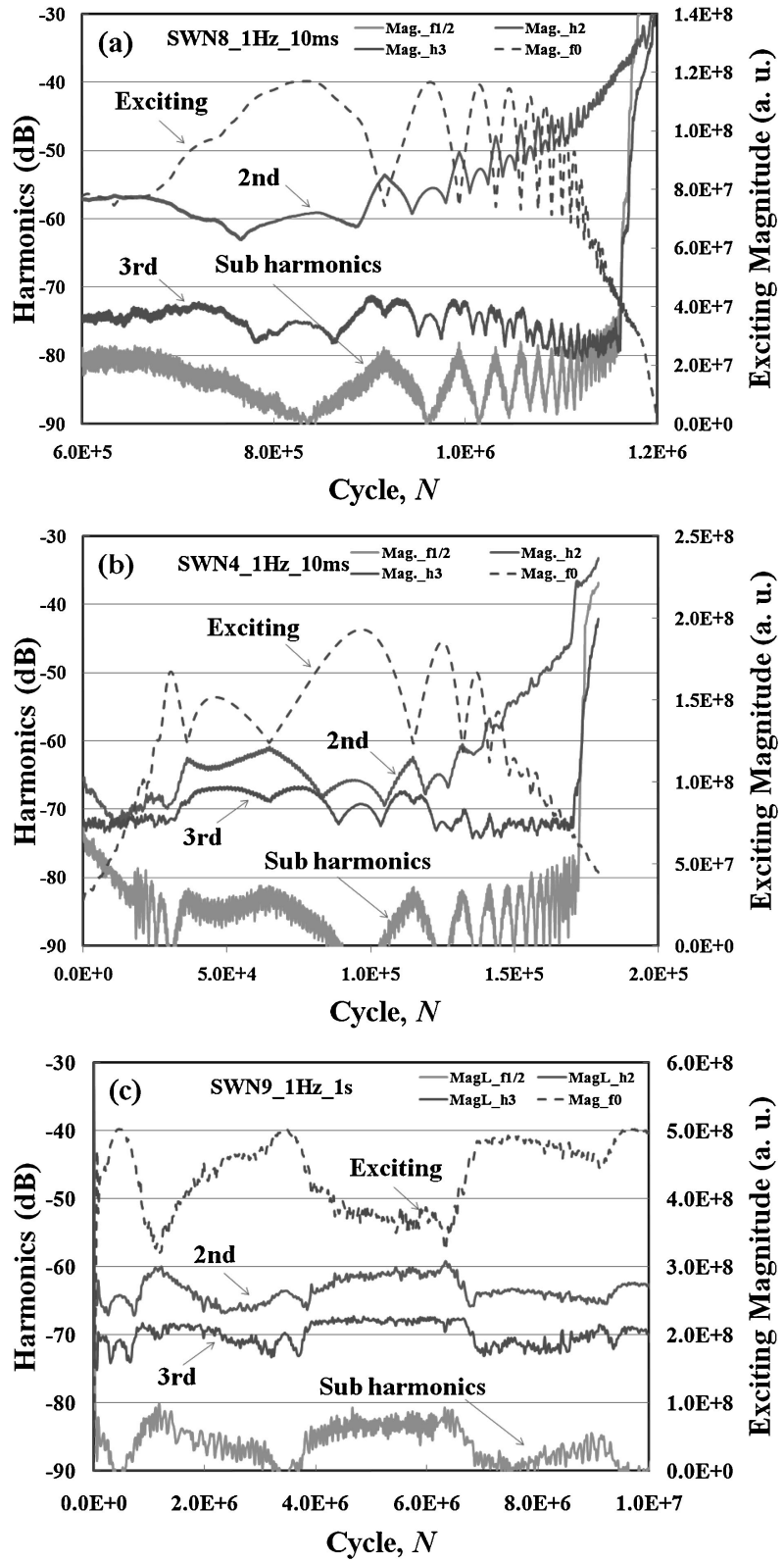

Fig. 5 Magnitude of resonant frequency and nonlinear ultrasonic, 2nd harmonics 3rd harmonics, sub harmonics during ultrasonic fatigue testing, Type I (a), Type II (b), Type III (c).

(b), Type III (c). The second harmonic, the third harmonic, and the sub harmonic with a half of the exciting frequency are adopted as the parameters of nonlinear ultrasonic. The decrease in the exciting magnitude was not observed in Type III of the unbroken specimen. Meanwhile, the substantial change of nonlinear ultrasonic parameters was not seen. On the other hand, the rise of the exciting magnitude was observed after the middle stage of the fatigue cycles in Type I and II of the broken specimen. Then a few peak and dip of that observed, the change in the cycle shortened near the breaking. The second harmonic in Type I and II increased gradually, and the third harmonic and sub harmonic had increased rapidly before breaking when the exciting magnitude starts to decrease.

\subsection{Acoustic emission analysis}

For observation of waveform during the fatigue testing, the change was not seen in Type III. On the contrary, the shape of the exciting wave had greatly warped immediately and there were pulse signals mixed in the final stage of the fatigue testing for Type I and II.

Figure 6 presents each stage of the detected waveforms with a long term period $(5 \mathrm{~ms})$ and a short term period ( $200 \mu \mathrm{s})$, respectively. Stage I in Fig. 6(a) is before the rise of the third harmonic in Type I and II, and is all term in Type III. Stage II and III in Figs. 6(b), (c) was observed only in Type I and II. In the stage II of Fig. 6(b), the modulation of waveform occurred and pulse signals mixed in the exciting waveform when the third harmonic rose. In the stage III of Fig. 6(c), it was just before breaking, waves with a half of the exciting frequency and pulse waves were observed. Up to the sixth harmonic were observed since Stage II. Therefore, a $100 \mathrm{kHz}$ high-pass filter (HPF) was processed to extract the pulse signals from the exciting waveform. Figure 7 gives the extracted waveform. This shape of waves was consistent with the acoustic emission (AE). Then, AE events were extracted by the threshold level of $100 \mathrm{mV}$ in Type I and II.

Figure 8 shows the magnitudes of exciting, sub harmonic, second harmonic, third harmonic and cumulative AE events as a function of fatigue cycle in Type I and II. The frequency resolution was $1 \mathrm{kHz}$ and the time step was $1 \mathrm{~ms}$ (each 20 cycles). In both of them, the second and third harmonics increased simultaneously when the exciting magnitude became unstable. Subsequently, the AE events started to increase. The AE events increased with the decrease of the exciting magnitude. Then, the magnitude of sub harmonics increased rapidly. Especially the exciting magnitude decreased rapidly at $1.203 \times 10^{6}$ cycles in Type I, and then it increased by $1.215 \times 10^{6}$ cycles before the final failure. This phenomenon was not seen in Type II.

Figure 9 offers the SEM morphology of fracture surface for Type I. It can be seen that the crack propagated at the notch tip while there was no striation pattern on the fracture surface. The fracture surface of the initial stage of crack propagation is a typical fatigue fracture surface with some facet depended on its microstructure. Some dimples can be seen after the middle stage of crack propagation, finally there are many dimple on the fracture surface. A similar fracture surface of the middle stage of crack propagation can be seen before the final failure. The crack stop once and it re-propagate again.

\section{Discussion}

For the ultrasonic fatigue testing, there are two problems. Firstly, there is a heating caused by the cyclic loading with a velocity two or three orders higher than that of the conventional mechanical fatigue testing. The heating sources are hysteresis to have roots in a dislocation movement, a local plastic deformation and an internal friction. Therefore, materials with low hysteresis energy can be applied to the ultrasonic fatigue testing. Secondly, there is a higher velocity of the ultrasonic wave compared with a dislocation velocity. The dislocation velocity under $1000 \mathrm{~m} / \mathrm{s}$ is relatively slower than that of the general ultrasonic wave. The high speed cyclic loading condition might have a possibility of dislocation movement arresting. 

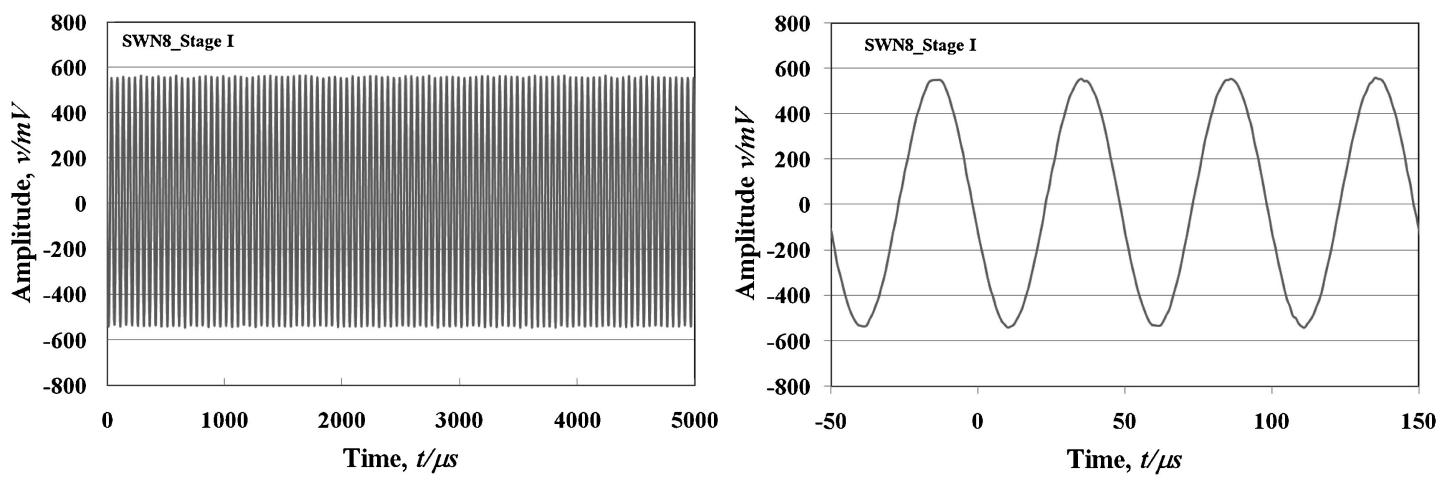

(a) Stage I
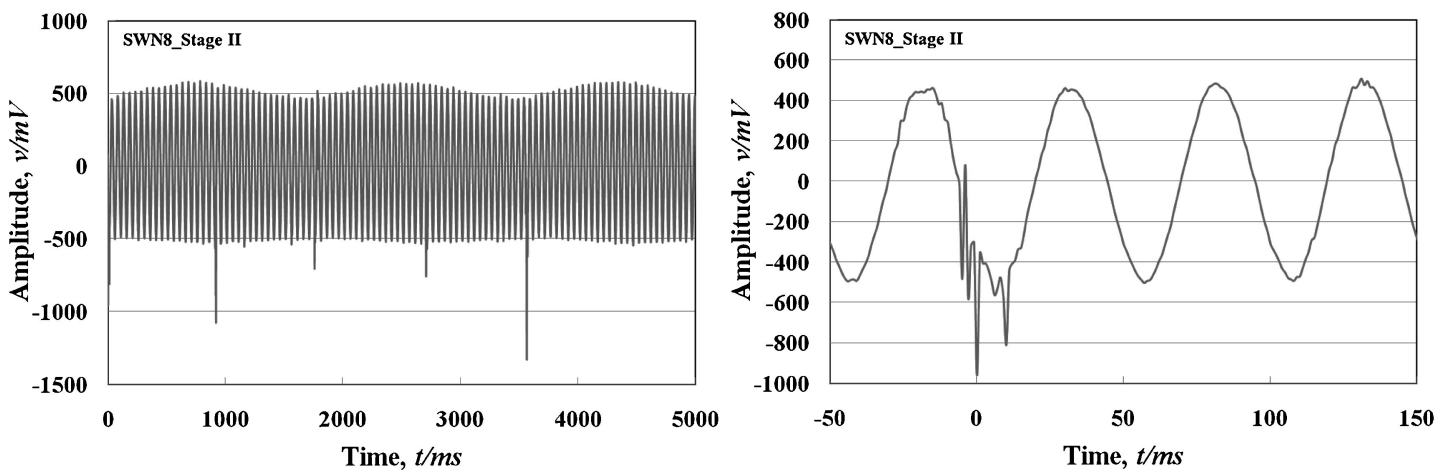

(b) Stage II
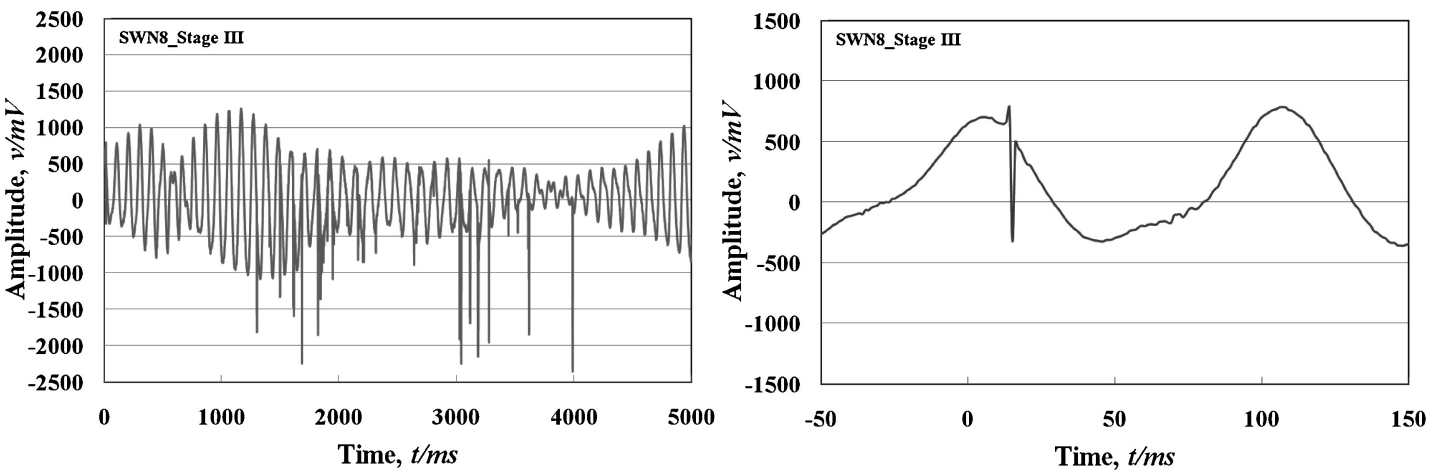

(c) Stage III

Fig. 6 Detected typical waveforms during ultrasonic fatigue testing, Stage I (a), Stage II (b), Stage III (c).

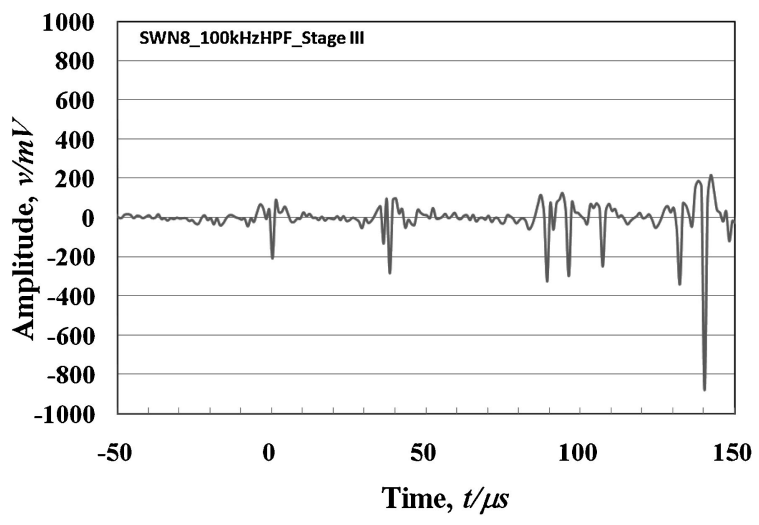

Fig. 7 Typical $100 \mathrm{kHz}$ HPF waveform of Stage III.

The harmonics of nonlinear ultrasonic is usually related to the dislocation movement. Previous experiments shows that based on analyses of the time domain deformation (disloca- tion motion mode) and the ultrasonic signal in frequency domain, it indicated that the even harmonics is related to the dislocation pileup and the odd harmonic is corresponding to the removing dislocation from pinning site. ${ }^{9)}$ For the all ultrasonic harmonics, the second and the third harmonics are often used due to their high intensities. In the conventional fatigue testing of a high hysteresis material, such as stainless steels, a parameter $\beta$ based on the harmonics ratio of each stage of damage progression specimens is good agreement with the hardness, amount of the plastic deformation and the fatigue life. ${ }^{3,4)}$

The present high-strength steel has a low hysteresis energy due to the low-temperature tempering leading to the low mobility of dislocation. ${ }^{2)}$ As a result, the decrease in the exciting magnitude and the increase in the second harmonic were observed from the number of cyclic loading of about $60 \%$ in the broken specimen of Type I and II, as shown in Fig. 4. It indicated that the dislocation density begins to 

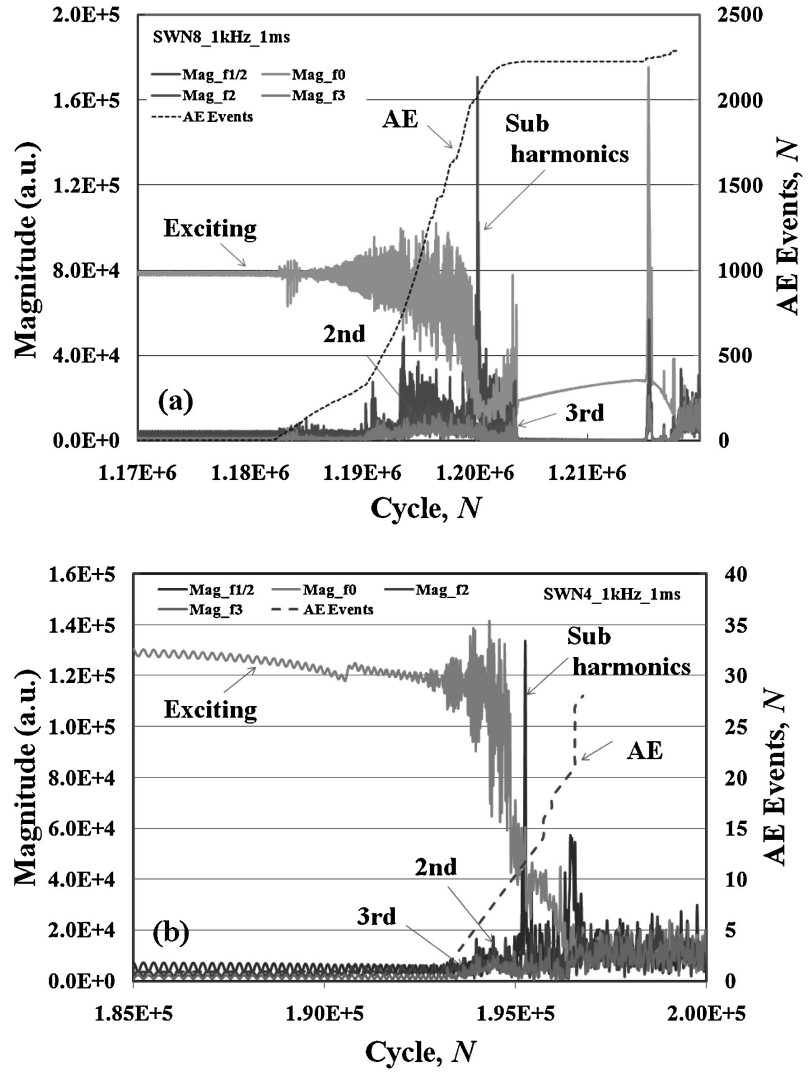

Fig. 8 Magnitude of resonant frequency and nonlinear ultrasonic (2nd harmonic 3rd harmonic, sub harmonic) and cumulative AE counts by each 20 cycles of $1 \mathrm{kHz}$ FFT resolution, Type I (a), Type II (b).

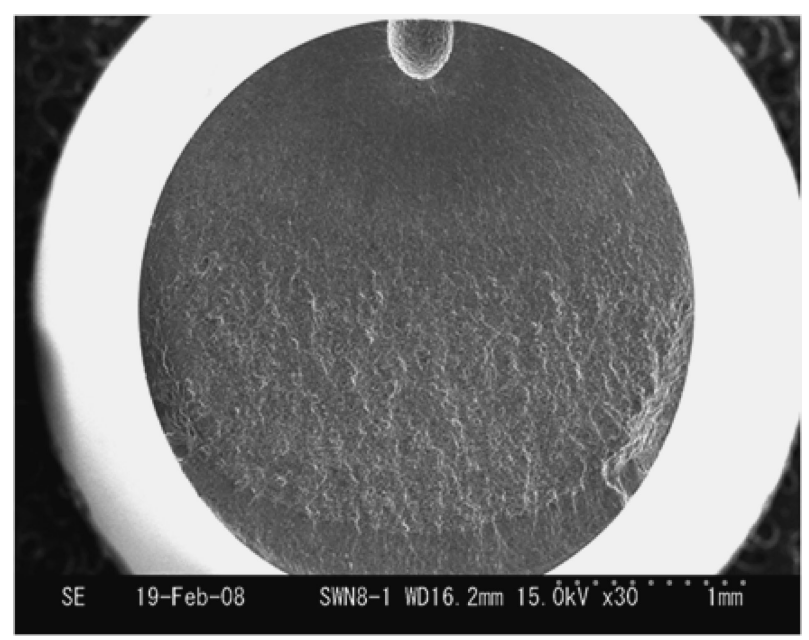

Fig. 9 Fracture surface of Type I by SEM.

increase in this stage. A periodic change in the exciting magnitude is caused by the ultrasonic testing machine to keep the applied stress. The third harmonic increased rapidly at the final stage of failure in Type I and II (as shown Fig. 5 and 8). The removing dislocation might be occurred at this stage. Therefore, the AE behavior was corresponding to the increase in the intensity of harmonic, as shown in Fig. 8. Besides, there was a rapidly increasing point of the sub harmonic after the AE generated. Because the sub harmonic is generated from hitting each crack surface and the $\mathrm{AE}$ is caused by the micro-crack generation and the main crack propagation. Consequently, the main crack propagated with creating new crack surface and the dislocation pileup and removing continuously. The fracture surface of Type I (in Fig. 9) is consistent with exciting and nonlinear ultrasonic phenomena as shown in Fig. 8. The technique of short time FFT, which is the frequency resolution of $1 \mathrm{kHz}$ and the time step of $1 \mathrm{~ms}$ ( 20 cycles), could be used to detect the phenomena of fatigue crack propagation, such as crack initiation, propagation, arrest and re-propagation under the cyclic loading. Also the technique of long time FFT, which is the frequency resolution of $1 \mathrm{~Hz}$ and the time step of $10 \mathrm{~ms}$ (200 cycles), could be used to detect he phenomena of dislocation such as pileup and removing before crack nucleation.

According to above analyses, it can be concluded that the nonlinear ultrasonic and AE signal analysis were effective monitoring tool for fatigue damage progression in the ultrasonic fatigue testing with measuring vibration by using wideband LDV.

\section{Conclusions}

In-situ observation of the fatigue damage progression behavior during the ultrasonic fatigue testing was carried out by using a wideband LDV. The evaluated signals are nonlinear ultrasonic, such as sub harmonic, harmonics and $\mathrm{AE}$ in the high strength steel specimens of the low cycle number of fatigue failure (Type I), the high cycle number of fatigue failure (Type II) and the non-breaking (Type III).

(1) For the strength of the nonlinear ultrasonic, the substantial change was not seen while testing in Type III of non-broken specimen. On the other hand, in both of Type I and Type II of the broken specimens, the second harmonic increased gradually, and the third harmonic and sub harmonic increased rapidly just before the broken.

(2) In Acoustic Emission signals through to high pass filter of $100 \mathrm{kHz}$, when the intensity of harmonics were increased in both of Type I and II, the number of AE events increased rapidly corresponding to the rising intensity of sub harmonic. Contrary the AE events did not detected in Type III.

(3) It can be concluded that non-linear ultrasonic and $\mathrm{AE}$ signal analysis were effective monitoring tool for fatigue damage progression in ultrasonic fatigue testing with measuring vibration by using wideband LDV.

\section{REFERENCES}

1) Y. Furuya, S. Matsuoka and T. Abe: Electric Furnace Steel 75 (2004) 55-60.

2) Y. Furuya, S. Matsuoka, T. Abe and K. Yamaguchi: Trans. Jpn. Soc. Mech. Eng. A 68 (2002) 477-83.

3) J. H. Cantrell and W. T. Yost: J. Appl. Phys. 81 (1997) 2957-2962.

4) J. H. Cantrell: J. Appl. Phys. 100 (2006) 063508-1-7.

5) K. Yamanaka, Y. Ohara, S. Yamamoto and T. Mihara: J. JSNDI 56 (2007) 280-285.

6) M. Shiwa, S. Carpenter and T. Kishi: J. Compo. Mater. 30 (1996) 2019-2041.

7) M. Shiwa, Y. Furuya, H. Yamawaki, K. Ito and M. Enoki: Rev. Quantitative Nondestructive Evaluation 28B (2009) 1450-1457.

8) K. Ito and M. Enoki: Mater. Trans. 48 (2007) 1221-1226.

9) T. Imanaka: J. JSNDI 56 (2007) 286-291. 